\title{
MicroRNA-mediated switching of chromatin-remodelling complexes in neural development
}

\author{
Andrew S. Yoo ${ }^{1,2,3}$, Brett T. StaahI ${ }^{1,2}$, Lei Chen ${ }^{1,2,3}$, and Gerald R. Crabtree 1,2,3 $^{1,2}$ \\ ${ }^{1}$ Howard Hughes Medical Institute, Stanford University, Stanford, California 94305, USA \\ 2 Department of Developmental Biology, Stanford University, Stanford, California 94305, USA \\ ${ }^{3}$ Department of Pathology, Stanford University, Stanford, California 94305, USA
}

\section{Abstract}

One of the most distinctive steps in the development of the vertebrate nervous system occurs at mitotic exit when cells lose multi-potency and begin to develop stable connections that will persist for a lifetime ${ }^{1,2}$. This transition is accompanied by a switch in ATP-dependent chromatinremodelling mechanisms that appears to coincide with the final mitotic division of neurons. This switch involves the exchange of the BAF53a (also known as ACTL6a) and BAF45a (PHF10) subunits within Swi/Snf-like neural-progenitor-specific BAF (npBAF) complexes for the homologous BAF53b (ACTL6b) and BAF45b (DPF1) subunits within neuron-specific BAF (nBAF) complexes in post-mitotic neurons. The subunits of the npBAF complex are essential for neural-progenitor proliferation, and mice with reduced dosage for the genes encoding its subunits have defects in neural-tube closure similar to those in human spina bifida ${ }^{3}$, one of the most serious congenital birth defects. In contrast, BAF53b and the nBAF complex are essential for an evolutionarily conserved program of post-mitotic neural development and dendritic morphogenesis ${ }^{4,5}$. Here we show that this essential transition is mediated by repression of BAF53a by miR-9* and miR-124. We find that BAF53a repression is mediated by sequences in the $3^{\prime}$ untranslated region corresponding to the recognition sites for miR-9* and miR-124, which are selectively expressed in post-mitotic neurons. Mutation of these sites led to persistent expression of BAF53a and defective activity-dependent dendritic outgrowth in neurons. In addition, overexpression of miR-9* and miR-124 in neural progenitors caused reduced proliferation. Previous studies have indicated that miR-9* and miR-124 are repressed by the repressor-element-1-silencing transcription factor (REST, also known as NRSF) ${ }^{6}$. Indeed, expression of REST in post-mitotic neurons led to derepression of BAF53a, indicating that RESTmediated repression of microRNAs directs the essential switch of chromatin regulatory complexes.

The ATP-dependent chromatin-remodelling complexes, typified by the yeast Swi/Snf complex, regulate chromatin assembly and accessibility ${ }^{7,8}$. The mammalian genome encodes nearly 30 different Swi2/Snf2-like ATPases, two of which, BRG1 and BRM are alternative subunits in complexes of 11 subunits termed BAF or mammalian SWI/SNF $(\mathrm{mSWI} / \mathrm{SNF})^{3,4,9-11}$. To understand the essential switch in subunit composition of these

Correspondence and requests for materials should be addressed to G.R.C. (crabtree@stanford.edu).

Supplementary Information is linked to the online version of the paper at www.nature.com/nature.

Author Contributions A.S.Y. and G.R.C. generated the hypotheses, designed experiments and wrote the manuscript. A.S.Y. performed experiments and generated Figs 1, 2, 3 and 4 and supplementary data. B.T.S. and A.S.Y. performed experiments for Figs 3 and 4. L.C. performed the pronuclear injections used in generating transgenic mouse embryos.

Author Information Reprints and permissions information is available at www.nature.com/reprints. 
complexes during neural development, we examined 180 kilobases $(\mathrm{kb})$ around the BAF53a gene for transcriptional regulatory regions by replacing the first exon of BAF53a at the start codon with a destabilized nuclear enhanced green fluorescent protein (d2nucEGFP) in a BAF53a-containing bacterial artificial chromosome (BAC). The d2nucEGFP is followed by the stop codon and a $3^{\prime}$ untranslated region (UTR) so that EGFP expression reflects BAF53a transcription (Fig. 1a, diagram). The BAF53a BAC reporter with a SV40 3' UTR gave persistent expression of EGFP in post-mitotic neurons in the neural tube (Fig. 1a, lower-left panel) and, hence, failed to mimic the expression pattern of the endogenous gene (Fig. 1b). However, when the $3^{\prime}$ UTR of BAF53a was used in place of the SV40 UTR, the d2nucEGFP reporter faithfully reproduced the downregulation of the endogenous BAF53a gene in post-mitotic neurons (Fig. 1a, lower-right panel, and Supplementary Fig. 1), implicating the evolutionarily conserved 3' UTR (Supplementary Fig. 1) in post-mitotic suppression of BAF53a.

The 3' UTR of BAF53a has predicted binding sites for microRNAs (miRNAs) miR-9, miR-9* and miR-124 (refs 12, 13; see Fig. 2a and Supplementary Fig. 2), small, 20-22-basepair (bp), non-coding RNAs that inhibit expression of their target genes by transcript degradation or translation inhibition ${ }^{14}$. These miRNAs are enriched in neurons ${ }^{15}$ and mouse brain tissues ${ }^{16}$. There appeared to be three sites for miR-9* and single sites for miR-9 and miR-124 (Fig. 2a and Supplementary Fig. 2). Short, 6-bp, sequences complementary to the $5^{\prime}$ ends of miRNAs are important for target recognition by miRNAs ${ }^{17,18}$. To determine whether the predicted binding sites for miRNAs are responsible for BAF53a repression, we inserted point mutations into the 3' UTR targeting sequences for miR-9, miR-9* and miR-124 (Fig. 2b, left panel). The mutant BAF53a 3' UTR failed to repress its reporter in post-mitotic neurons (Fig. 2b and Supplementary Fig. 3), indicating that the predicted binding sites in the $3^{\prime}$ UTR mediate BAF53a downregulation.

To distinguish the relative roles of the different miRNAs targeting BAF53a, we designed a construct to 'sense' and quantify the miRNA-mediated repression of the reporter through the $3^{\prime}$ UTR of BAF53a. This sensor construct contains the d2nucEGFP with test 3' UTRs and tomato fluorescent protein (nuc mTom) with the invariant SV40 3' UTR (Supplementary Fig. 4). Stable cell lines containing miR-9, miR-9* and miR-124 overexpression and sensor constructs were established (Supplementary Fig. 4). The function of 3' UTRs in EGFP expression was scored as the ratio of EGFP/Tomato signals in individual cells. When the BAF53a 3' UTR was tested, EGFP expression was significantly reduced relative to EGFP expression with SV40 3' UTR (Fig. 2c). Mutating the miR-9* and miR-124 sites abolished the downregulation of the EGFP signal, and removal of the miR-9 site gave no additional derepression (Fig. 2c). Mutating only the miR-9* sites or the miR-124 site was not sufficient to abolish the repression of the EGFP signal. These results suggest that miR-9* and miR-124 are both required to inhibit BAF53a expression. Furthermore, in situ analysis showed that miR-9* was expressed mostly in post-mitotic neurons in the neural tube of E11.5 embryos (Fig. 2d). Published studies show that miR-124 was expressed in a nearly identical pattern to miR-9* (refs ${ }^{19-21}$ ), indicating that both miR-9* and miR-124 are expressed just before BAF53a repression in post-mitotic neurons.

MicroRNAs typically target multiple genes ${ }^{22}$. To define the contribution of miRNAmediated repression of BAF53a to neural development, we prepared transgenic embryos expressing the BAC containing mutations in the 3' UTR of BAF53a. In contrast to the wildtype BAC-transgenic embryos (Fig. 3a), embryos containing the mutant BAC transgene displayed a loss of the clear boundary of BAF53a downregulation, and instead showed irregular expansion of BAF53a expression into $\beta$-tubulin-III-positive neurons (Fig. 3b). Because BAF53a and BAF53b are expressed in a mutually exclusive pattern (Fig. 1b), we then investigated whether BAF53b expression would be affected by the prolonged 
expression of BAF53a. Embryos transgenic with the wild-type BAF53a BAC showed strong and specific expression of BAF53b in $\beta$-tubulin-III-positive neurons (Fig. 3c). However, continued expression of BAF53a in neurons repressed BAF53b expression (Fig. 3d, e). These results suggest that BAF53a directly or indirectly antagonizes BAF53b expression, and provide a possible explanation of how the mutually exclusive BAF switching is achieved during neural development.

Because BAF53b is essential for dendritic morphogenesis ${ }^{4,5}$, this result suggests that miRNA-mediated repression of BAF53a is essential for the onset of post-mitotic neuronal function. We tested this hypothesis by overexpressing BAF53a in cultured hippocampal neurons and assayed activity-dependent dendritic outgrowth (stimulated by $\mathrm{KCl}$ treatment ${ }^{4}$ ). Persistent expression of BAF53a in neurons inhibited stimulated dendritic outgrowth (Fig. $3 \mathrm{f}$ ), elucidating the importance of BAF53a switching in neurons. We then tested the contribution of miRNA-mediated regulation of BAF53a in dendritic outgrowth. When BAF53a was expressed with the SV40 3' UTR, we observed strong inhibition of dendritic outgrowth in neurons; expression with the wild-type BAF53a 3' UTR did not produce such inhibition (Fig. 3g). The dendritic phenotype reappeared when we mutated the binding sites for miR-9* and miR-124 in the BAF53a 3' UTR (Fig. 3g). These results underline the essential role of miR-9*-and miR-124-mediated BAF53a repression in dendritic outgrowth.

To elucidate further the function of miR-9* and miR-124, we created transgenic embryos in which miR-9* and -124 were ectopically expressed under the control of the nestin promoter in neural progenitors (Fig. 4a). Control transgenic embryos in which the nestin promoter drove EGFP expression only showed the expected co-localization between EGFP-positive cells and BAF53a expression. When the nestin promoter drove expression of miR-9* and miR-124 ectopically in progenitors, transgenic embryos showed significantly reduced expression of BAF53a in progenitors (Fig. 4b, top-right graph). This reduction in BAF53a expression was probably not due to precocious differentiation of the progenitors into neurons, because the miR-9* and miR-124 expressing progenitors did not show staining for BAF53b (Supplementary Fig. 5).

As BAF53a has previously been shown to promote proliferation of progenitors ${ }^{3}$, we asked whether the reduction in BAF53a expression would be associated with the proliferation state of progenitors. As expected, control transgenic embryos showed complete co-localization between $\mathrm{K}_{\mathrm{i}}-67$ (a marker for proliferating cells) and EGFP-positive progenitors (Fig. 4b). We found that overexpressing miR-9* and miR-124 hindered the proliferation of progenitors, evidenced by the significantly reduced expression of $\mathrm{K}_{\mathrm{i}}-67$ in EGFP-positive progenitors (Fig. 4b). This proliferation was significantly restored when miRNA-resistant BAF53a (BAF53a ${ }^{\Delta \Delta}$ ) was expressed with miR-9* and miR-124 (Fig. 4b). The results are consistent with the previous finding that one of the functions of BAF53a and other components of the npBAF complex in progenitors is to promote proliferation, and suggest a role for miR-9* and miR-124 in combination to prevent proliferation in post-mitotic neurons by removing BAF53a expression. We wondered whether prolonging BAF53a expression in already committed neurons would be sufficient to trigger re-entry into the cell cycle in neurons. In mutant BAF53a BAC-transgenic embryos, BAF53a expression was extended into neurons (Fig. 3b). However, $\mathrm{K}_{\mathrm{i}}-67$ expression eventually disappeared in the neuronal zone in these embryos, suggesting that continued BAF53a expression in committed neurons was not sufficient to cause neurons to re-enter the cell cycle (Supplementary Fig. 6).

Consistent with this finding, expressing BAF53a in neuronal cultures was insufficient to reactivate proliferation (data not shown).

The miRNAs miR-9* and miR-124 have been shown to be targets of REST ${ }^{6}$. REST, with its co-repressor, restricts the activation of neuronal genes to neurons by silencing its target 
genes in non-neuronal cells; REST activity is reduced as progenitors differentiate into neurons, allowing the expression of neuronal genes ${ }^{23-28}$. All three loci of miR-9* and miR-124 contain REST-binding sites, which inhibit miR-9* and miR-124 expression ${ }^{6}$, suggesting that REST expression would activate BAF53a in post-mitotic neurons. Neurons transfected with an REST-expression plasmid reactivated BAF53a expression, which contrasted with the absence of BAF53a expression in control-transfected neurons (Fig. 4c). We reasoned that this BAF53a expression in neurons should be suppressed if we cointroduced a plasmid that overexpresses miR-9* and miR-124 when driven by a promoter whose activity is independent of REST. Indeed, co-transfection of miR-9* and miR-124 along with REST-expression plasmids suppressed BAF53a reactivation in neurons (Fig. 4c), suggesting an antagonism between REST and miRNAs in repressing BAF53a in postmitotic neurons.

Our studies indicate that the mechanism underlying the essential switch in subunit composition of Swi/Snf-like BAF complexes involves miRNA-mediated repression of BAF53a as neural progenitors differentiate into neurons (Fig. 4d). The repression is accomplished through the $3^{\prime}$ UTR of BAF53a and mediated by the simultaneous activities of miR-9* and miR-124. REST participates in this switch by repressing miR-9* and miR-124, thereby permitting BAF53a expression in neural progenitors. In neurons, the absence of REST allows the expression of miR-9* and miR-124 to repress BAF53a (Fig. 4d). Repression of BAF53a allows BAF53b expression, which is critical for dendritic development ${ }^{4,5}$. Failure of miRNA-mediated repression of BAF53a results in reduced BAF53b expression and defective dendritic outgrowth (Fig. 4d). Because repression of BAF53a reduces progenitor proliferation, miRNA-mediated repression of BAF53a may be essential in regulating the number of cell divisions in the vertebrate nervous system. Mice with reduced BAF155 and BRG1 levels have defects in neural-tube closure similar to spina bifida in humans ${ }^{3,29,30}$, indicating a genetically dominant role of npBAF in progenitor proliferation. Thus, studies of the genes involved in the npBAF-to-nBAF switch might yield insights into the pathogenesis of spina bifida and perhaps also the mechanisms controlling the number cell divisions in the central nervous system.

\section{METHODS SUMMARY}

\section{Transgenic mouse embryos}

We made BAC-based reporters by recombineering using galK-positive and -negative selections. Detailed protocols for constructing BAC reporters and other related transgene constructs are provided in the full Methods. Transgenic mouse embryos were generated by pronuclear injection of fertilized oocytes from CB6-F1 mice, and analysed 11.5 days after fertilization in cryostat sections immunostained with appropriate antibodies.

\section{Tissue culture}

We cultured E18.5 hippocampal neurons on coverslips coated with poly-L-ornithine, fibronectin and laminin in 24-well plates for four days in vitro (div), and transfected with appropriate plasmids after 4 div. Cells were fixed and stained $48 \mathrm{~h}$ later, after 6 div. Immunocytochemistry was done with affinity-purified BAF53a and Tuj-1 (BD Biosciences) primary antibodies and goat anti-rabbit or mouse secondary antibodies (Invitrogen) with DAPI (Vector) DNA staining. Detailed protocols are provided in the full Methods. 


\title{
METHODS
}

\section{Recombineering of BAC containing BAF53a and mutagenesis}

BAC (RP23-370017, approximately $180 \mathrm{~kb}$ around BAF53a) was recombined at the first exon of BAF53a with d2nucEGFP, using the galK-positive and -negative selection method as previously described ${ }^{31}$. SW106 cells were transformed with RP23-370O17, selected and transformed with galK PCR product amplified by primers with 50-bp arms homologous to the sites to be recombined: galK FWD,

TGTTATCTTTCGTCCGGTAGTCTTCGGCCAGTCCCCGCCAGACAGTAGCC CCTGTTGACAATTAATCATCGGCA; galK REV, GGCCGGTCGTTCCGGTCCGCACGCCCCGAGGCGCCGCTGCGCTCACTCACTCAG CACTGTCCTGCTCCTT.

\begin{abstract}
Recombination was performed by heat-shocking the transformed SW106 cells, and colonies containing the recombined products were selected on minimal agarose plates plus galactose. The galK-containing RP23-370O17 was then recombined to replace galK with $\mathrm{d} 2$ nucEGFP gene by transforming SW106 cells with PCR product (below) of d2nucEGFP/3' UTR and homology arms. Different versions of $3^{\prime}$ UTRs were subcloned into a d2nucEGFPcontaining plasmid, and used as templates for PCR amplification. d2nucEGFP FWD, TGTTATCTTTCGTCCGGTAGTCTTCGGCCAGTCCCCGCCAGACAGTAGCC ATGGTGCACGTGGATCCAAAAAAG; d2nucEGFP REV (for SV40 UTR): GGCCGGTCGTTCCGGTCCGCACGCCCCGAGGCGCCGCTGCGCTCACTCAC ATCGCCCTGATAGACGGTTTTTCG; d2nucEGFP REV (for BAF53a UTR): GGCCGGTCGTTCCGGTCCGCACGCCCCGAGGCGCCGCTGCGCTCACTCACGGGA AGGATCTGAGGAGGGCTCAG.
\end{abstract}

Point mutations in BAF53a 3' UTR were generated by QuikChange mutagenesis (Stratagene) using the following primers (bold fonts denote mutated base pairs): miR-9* site 1, CACCTCAACGTCTGTACGAATAGTACACTCAGGAA; miR-9* site2-1, CCACTTAAATTTTTTAAGCGAATAACTGGCTCTATAAATTAAATG; miR-9* site2-2, CTTAAATTTTTTAAGCGAATAAGACCGCCTATAAATTAAATGAG; miR-9* site3, GAATGTCTATTTTCTCTAAATATTTTCGAATCAGTAAGTACTCTCC; miR-124 site, GTACAAGTTACTGCCTAACGAAACTACCTTAGGCTACAAAAC; miR-9 site, TGTACGAATAGTACACACTCCTAAAGATGACCATCTTTTG.

SW106 cells transformed with d2nucEGFP PCR product were grown on 2-deoxy-Dgalactose-containing minimal plates for negative selection. Colonies with correctly recombined BACs were identified by PCR using primers surrounding the recombined $5^{\prime}$ and $3^{\prime}$ junctions. BAC DNA was prepared from the isolated colonies, rechecked by PCR and further confirmed by sequencing.

BAF53a BAC containing mutations in the 3' UTR of BAF53a was generated similarly by recombining the 3'UTR of BAF53a with the PCR product of mutant 3' UTR plus homology arms using the following primers: BAF53a UTR H1 galK FWD,

AGGAATATGAAGAAGGAGGGAAGCAGTGTGTAGAAAGAAAATGCCCTTGACCT GTTGACAATTAATCATCGGCA; BAF53a UTR H2 galK REV, CAGCAAAGTCAGTTTGGAAATGCTGTTCTCACTAGTGTTAAACTGTGAGGTCAG CACTGTCCTGCTCCTT; BAF53a UTR H1 FWD, AGG AATATGAAGAAGGAGGGAAGCAGTGTGTAGAAAGAAAATGCCCTTGAGGGCTC CACCCTGCCTGCC; BAF53a UTR H2 REV, CAGCAAAGTCAGTTTGGAAATGCTGTTCTCACTAGTGTTAAACTGTGAGGGGGA AGGATCTGAGGAGGGCTCAG. 


\section{Transgenic embryos}

All BAC-transgenic embryos were generated by pronuclear injections. More than three E11.5 embryos were examined for each BAC-reporter transgene.

\section{Data analysis}

Images for embryonic sections were captured using a Leica DM5000B microscope with Leica Application Suite (LAS) Advanced Fluorescence 1.8.0. Intensity measurements for BAF53a expression in REST expression (Fig. 4c) were performed with LAS 1.8.0. Images for stable Chinese hamster ovary lines containing sensor reporters were made using a Leica DMI4000B microscope with LAS 2.8.1. Image intensity analyses for sensor experiments (Fig. 2c) were performed with METAMORPH (version 7, Molecular Devices) using the images captured with the same exposure parameters. Co-localization analyses for Fig. $4 \mathrm{~b}$ were also performed using METAMORPH.

\section{Fluorescent in situ hybridization}

Fluorescent in situ analysis was performed using the fluorescein-labelled locked nucleic acid (LNA) oligonucleotide for miR-9* (Exiqon). Detailed protocols for LNA oligonucleotide in situ staining are provided on the manufacture's website (www.exiqon.com). The same sections were also stained for $\beta$-tubulin III using Tuj-1 antibody (BD Biosciences).

\section{MicroRNA cluster construct}

A cluster of miR-9* and miR-124 precursors was made by amplifying approximately 300 base pairs around mmu-miR-9*-3 and mmu-miR-124-2, which were together cloned into pSilencer (Ambion). mmu-miR-9*-3 precursor was amplified by the following primers containing appropriate restriction sites (not shown): mmu-miR-9-3 OX FWD, TGTCGGTCCCCTCTGGCTCTC; mmu-miR-9-3 OX REV, AGGAGAGCCGAGGTCGGATACC. mmu-miR-124-2 precursor was amplified using the following primers containing appropriate restriction sites: mmu-miR-124-2 OX FWD, AGGTGGGAGTACTGCTCAGAGCTA; mmu-miR-124-2 OX REV: GTGTCGCACAGGATGGGTTC.

The ability of the cluster construct to generate mature miR-9* and miR-124 was assayed by quantitative real-time PCR using a TaqMan miR-9* and miR-124 microRNA assay kit (Applied Biosystems). RNA was extracted from COS7 cells transfected with the miR-9*-124 overexpression construct using a mirVana miRNA isolation kit (Ambion). The same cluster of miR-9* and miR-124 was used for the constructs containing nestin promoters (Fig. 4a). Proliferation of progenitors in nestin p/miR-9*-124-transgenic embryos was assayed by immunostaining with Ki-67 antibody (BD Biosciences).

\section{Hippocampal neuronal culture and REST overexpression experiments}

The pActin-IRES-EGFP plasmid was constructed by inserting the chicken $\beta$-actin promoter followed by the IRES-EGFP module from the pIRES2-EGFP plasmid (Clontech) into pUC19 backbone. The pActin-REST-IRES-EGFP construct was generated by cloning the coding region of mouse REST gene into the pActin-IRES-EGFP vector. E18.5 murine hippocampal cells were cultured as previously described ${ }^{32}$. Dissociated cells were cultured on coverslips coated with poly-L-ornithine, fibronectin and laminin in 24-well plates. AraC was added after 1 div. Culture media contained DMEM/F12 with putrescine, 2mercaptoethanol, transferrin, insulin, selenium, progesterone, MEM vitamin additive and 5\% FBS. After 4 div, cells were transfected with GFP or other constructs using Lipofectamine (Invitrogen) as per the manufacturer's suggestions. Each transfection had 0.6 $\mu \mathrm{g}$ total DNA: pCMV-Bcl2 (to prevent occasional apoptosis of neurons associated with 
transfection), pActin-EGFP (control) or pActin-REST-EGFP, with or without pSilmir-9*-124. Cells were fixed (4\% PFA) and stained $48 \mathrm{~h}$ later, after 6 div.

Immunocytochemistry was done with affinity-purified BAF53a and Tuj-1 (BD Biosciences) primary antibodies and goat anti-rabbit or mouse secondary antibodies (Invitrogen) with DAPI (Vector) DNA staining.

\section{Dendritic outgrowth analysis}

Hippocampal neurons were prepared from P0 mouse brains and transfected with BAF53a overexpression constructs on day one. Activity-dependent dendritic outgrowth analysis was performed as described ${ }^{4}$. Briefly, after 4 div, cells were treated with $\mathrm{KCl}(30 \mathrm{mM})$ in half of the cultures. Cells were fixed and stained for GFP (Molecular Probes) and MAP2 (to mark dendrites; Sigma-Aldrich) after 5 div. A GFP construct was co-transfected to visualize transfected neurons for the experiments shown in Fig. 3f, g. The total dendritic length was measured from dendritic structures defined by GFP and MAP2 signals in individual neurons using the neurite outgrowth extension of METAMORPH.

\section{Prediction of miRNAs targeting BAF53a}

We used PICTAR ${ }^{12}$ and RNA22 ${ }^{13}$ to predict miRNAs that target BAF53a 3' UTR. Because BAF53a downregulation is specific for neurons, we sought miRNAs enriched in neurons and the brain ${ }^{15,16}$. miR-9* was the highest-scored miRNA when the BAF53a 3' UTR was searched in PICTAR. We further examined the $3^{\prime}$ UTR for additional sites for other miRNAs enriched in the brain using RNA22 (number of unpaired bases allowed, 1; seed/ nucleus ratio, 7; minimum number of paired bases in heteroduplex, 14; maximum folding energy, $-18 \mathrm{Kcal} \mathrm{mol}^{-1}$ ) and identified the binding sites for miR-124 and miR-9 as outlined in Fig. 2.

\section{Supplementary Material}

Refer to Web version on PubMed Central for supplementary material.

\section{Acknowledgments}

We thank J. I. Wu and J. Lessard for suggestions. We thank N. Copeland and N. Jenkins for providing us with the reagents for recombineering techniques. A.S.Y. is a fellow of Helen Hay Whitney Foundation. B.T.S. is supported by the Developmental and Neonatal Biology Training Program 2 T32 HD007249 from the US National Institutes of Health (NIH). This work was supported by grants from the Howard Hughes Medical Institute and the NIH, HD55391, AI060037 and NS046789, to G.R.C.

\section{References}

1. Noctor SC, Martinez-Cerdeno V, Ivic L, Kriegstein AR. Cortical neurons arise in symmetric and asymmetric division zones and migrate through specific phases. Nature Neurosci 2004;7:136-144. [PubMed: 14703572]

2. Temple S. The development of neural stem cells. Nature 2001;414:112-117. [PubMed: 11689956]

3. Lessard J, et al. An essential switch in subunit composition of a chromatin remodeling complex during neural development. Neuron 2007;55:201-215. [PubMed: 17640523]

4. $\mathrm{Wu}$ J, et al. Regulation of dendritic development by neuron-specific chromatin remodeling complexes. Neuron 2007;56:94-108. [PubMed: 17920018]

5. Parrish JZ, Kim MD, Jan LY, Jan YN. Genome-wide analyses identify transcription factors required for proper morphogenesis of Drosophila sensory neuron dendrites. Genes Dev 2006;20:820-835. [PubMed: 16547170]

6. Conaco C, Otto S, Han JJ, Mandel G. Reciprocal actions of REST and a microRNA promote neuronal identity. Proc Natl Acad Sci USA 2006;103:2422-2427. [PubMed: 16461918] 
7. Laurent BC, Treich I, Carlson M. The yeast SNF2/SWI2 protein has DNA-stimulated ATPase activity required for transcriptional activation. Genes Dev 1993;7:583-591. [PubMed: 8458575]

8. Peterson CL, Herskowitz I. Characterization of the yeast SWI1, SWI2, and SWI3 genes, which encode a global activator of transcription. Cell 1992;68:573-583. [PubMed: 1339306]

9. Khavari PA, Peterson CL, Tamkun JW, Mendel DB, Crabtree GR. BRG1 contains a conserved domain of the SWI2/SNF2 family necessary for normal mitotic growth and transcription. Nature 1993;366:170-174. [PubMed: 8232556]

10. Lemon B, Inouye C, King DS, Tjian R. Selectivity of chromatin-remodelling cofactors for ligandactivated transcription. Nature 2001;414:924-928. [PubMed: 11780067]

11. Wang W, et al. Diversity and specialization of mammalian SWI/SNF complexes. Genes Dev 1996;10:2117-2130. [PubMed: 8804307]

12. Krek A, et al. Combinatorial microRNA target predictions. Nature Genet 2005;37:495-500. [PubMed: 15806104]

13. Miranda KC, et al. A pattern-based method for the identification of microRNA binding sites and their corresponding heteroduplexes. Cell 2006;126:1203-1217. [PubMed: 16990141]

14. Carthew RW, Sontheimer EJ. Origins and mechanisms of miRNAs and siRNAs. Cell 2009;136:642-655. [PubMed: 19239886]

15. Krichevsky AM, Sonntag KC, Isacson O, Kosik KS. Specific microRNAs modulate embryonic stem cell-derived neurogenesis. Stem Cells 2006;24:857-864. [PubMed: 16357340]

16. Lagos-Quintana M, et al. Identification of tissue-specific microRNAs from mouse. Curr Biol 2002;12:735-739. [PubMed: 12007417]

17. Didiano D, Hobert O. Molecular architecture of a miRNA-regulated 3' UTR. RNA 2008;14:12971317. [PubMed: 18463285]

18. Filipowicz W, Bhattacharyya SN, Sonenberg N. Mechanisms of post-transcriptional regulation by microRNAs: are the answers in sight? Nature Rev Genet 2008;9:102-114. [PubMed: 18197166]

19. Cao X, Pfaff SL, Gage FH. A functional study of miR-124 in the developing neural tube. Genes Dev 2007;21:531-536. [PubMed: 17344415]

20. Makeyev EV, Zhang J, Carrasco MA, Maniatis T. The microRNA miR-124 promotes neuronal differentiation by triggering brain-specific alternative pre-mRNA splicing. Mol Cell 2007;27:435448. [PubMed: 17679093]

21. Visvanathan J, Lee S, Lee B, Lee JW, Lee SK. The microRNA miR-124 antagonizes the antineural REST/SCP1 pathway during embryonic CNS development. Genes Dev 2007;21:744-749. [PubMed: 17403776]

22. Flynt AS, Lai EC. Biological principles of microRNA-mediated regulation: shared themes amid diversity. Nature Rev Genet 2008;9:831-842. [PubMed: 18852696]

23. Lunyak VV, et al. Corepressor-dependent silencing of chromosomal regions encoding neuronal genes. Science 2002;298:1747-1752. [PubMed: 12399542]

24. Ballas N, Grunseich C, Lu DD, Speh JC, Mandel G. REST and its corepressors mediate plasticity of neuronal gene chromatin throughout neurogenesis. Cell 2005;121:645-657. [PubMed: 15907476]

25. Chen ZF, Paquette AJ, Anderson DJ. NRSF/REST is required in vivo for repression of multiple neuronal target genes during embryogenesis. Nature Genet 1998;20:136-142. [PubMed: 9771705]

26. Chong JA, et al. REST: a mammalian silencer protein that restricts sodium channel gene expression to neurons. Cell 1995;80:949-957. [PubMed: 7697725]

27. Schoenherr CJ, Anderson DJ. The neuron-restrictive silencer factor (NRSF): a coordinate repressor of multiple neuron-specific genes. Science 1995;267:1360-1363. [PubMed: 7871435]

28. Kuwabara T, Hsieh J, Nakashima K, Taira K, Gage FH. A small modulatory dsRNA specifies the fate of adult neural stem cells. Cell 2004;116:779-793. [PubMed: 15035981]

29. Bultman S, et al. A Brg1 null mutation in the mouse reveals functional differences among mammalian SWI/SNF complexes. Mol Cell 2000;6:1287-1295. [PubMed: 11163203]

30. Kim JK, et al. Srg3, a mouse homolog of yeast SWI3, is essential for early embryogenesis and involved in brain development. Mol Cell Biol 2001;21:7787-7795. [PubMed: 11604513] 
31. Warming S, Costantino N, Court DL, Jenkins NA, Copeland NG. Simple and highly efficient BAC recombineering using galK selection. Nucleic Acids Res 2005;33:e36. [PubMed: 15731329]

32. Graef IA, et al. L-type calcium channels and GSK-3 regulate the activity of NF-ATc4 in hippocampal neurons. Nature 1999;401:703-708. [PubMed: 10537109] 


\section{a}

BAC reporter with SV40 $3^{\prime}$ UTR
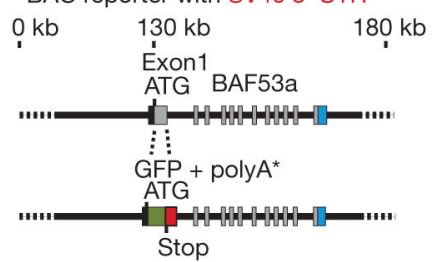

*d2nucEGFP + SV40 3' UTR, not drawn to scale

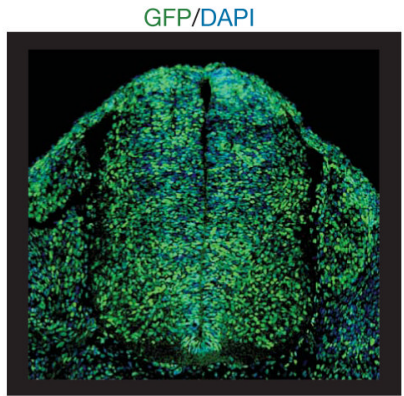

b

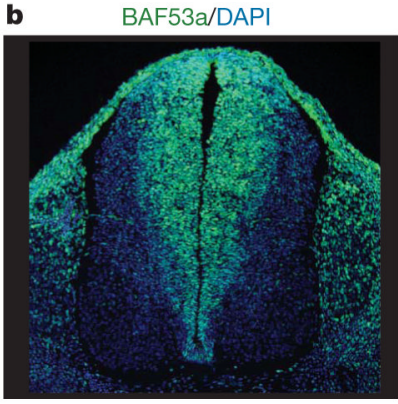

BAC reporter with BAF53a 3' UTR

$0 \mathrm{~kb} \quad 130 \mathrm{~kb} \quad 180 \mathrm{~kb}$

Éxon1 1 BAF53a

ATG BAF53a

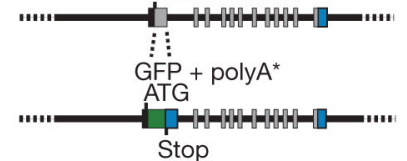

*d2nucEGFP + BAF53a 3' UTR,

not drawn to scale

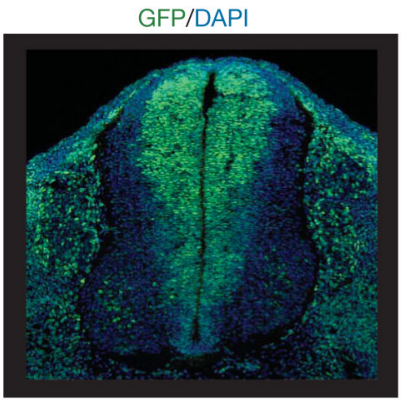

BAF53b/DAPI

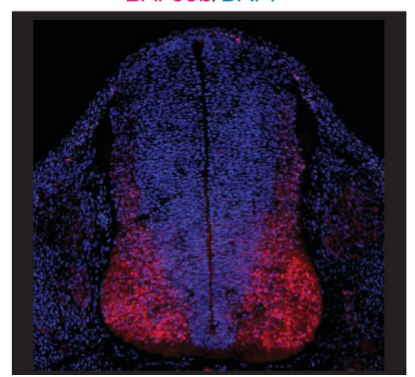

Figure 1. BAF53a repression is mediated by sequences within its $3^{\prime}$ UTR

a, Schematic diagrams of BAC clones of approximately $180 \mathrm{~kb}$ containing BAF53a and the BAC-based reporter constructs. Exon 1 from the ATG of BAF53a to the end of the exon was replaced by d2nucEGFP and 3' UTR. Whereas EGFP failed to be repressed in neurons with SV40 UTR (lower-left panel), the BAF53a 3' UTR allowed efficient downregulation of the reporter in neurons (lower-right panel). Photographs in the lower panels show transverse sections of the neural tubes of E11.5 transgenic embryos. DAPI, 4,6-diamidino-2phenylindole. b, BAF53a and BAF53b are expressed selectively in progenitors (green) and neurons (red), respectively, in the neural tubes of E11.5 embryos. 
a
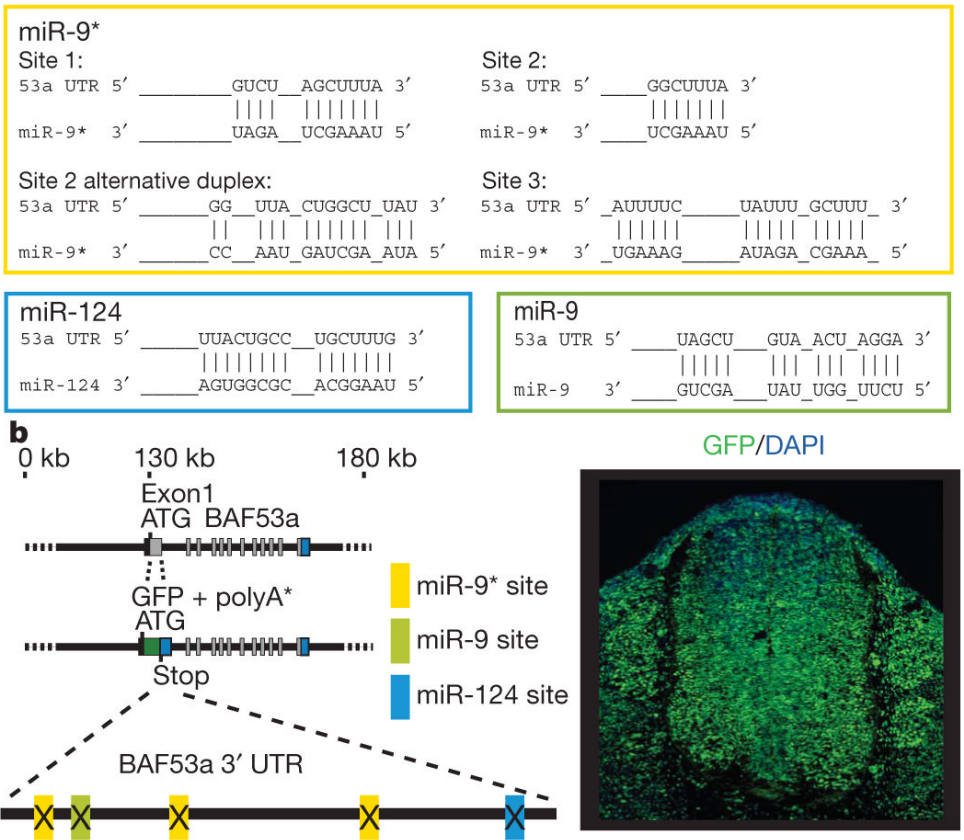

c
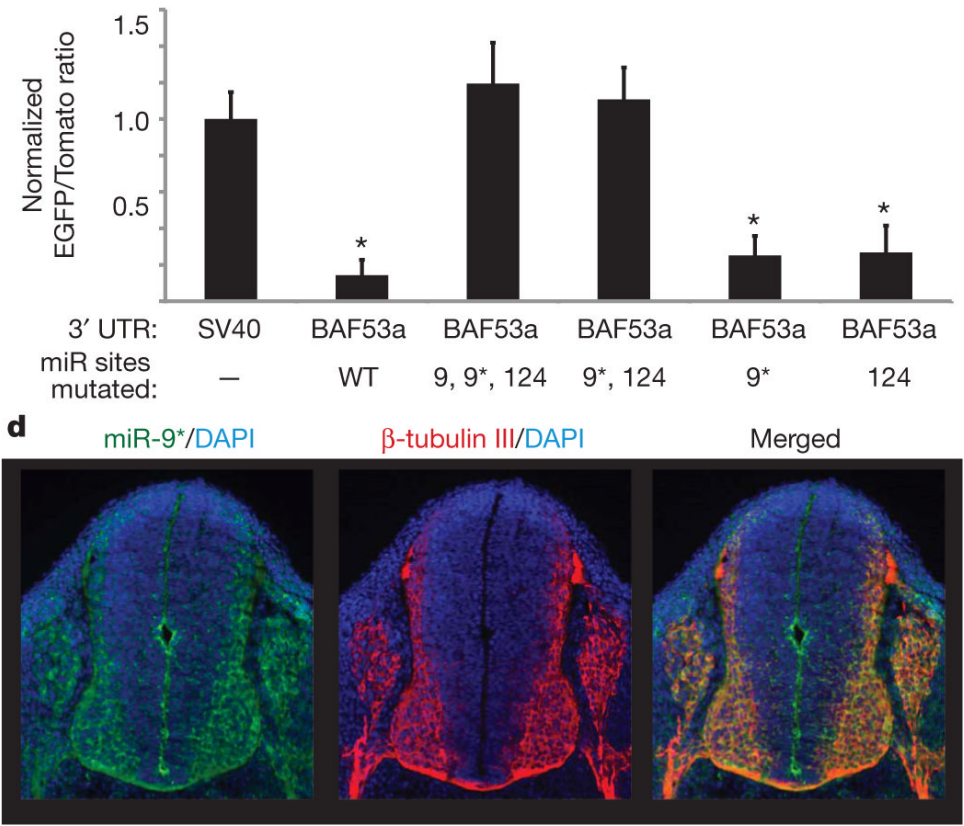

Figure 2. BAF53a is a target of miR-9* and miR-124

a, Predicted configurations of duplex formations between miRNAs and their target sites. b, Expression of BAF53a BAC reporter, containing mutations at the miRNA-binding sites in the $3^{\prime}$ UTR of d2nucEGFP. Point mutations were made to 4-6 bp predicted to bind to the 5' ends of miR-9, miR-9* and miR-124. The photograph shows a representative expression pattern seen in three individual transgenic lines. c, BAF53a 3' UTR sensor experiments in stable Chinese hamster ovary (CHO) cells expressing miR-9, mirR-9* and miR-124 and sensor reporter constructs. The quantification of sensor expression was expressed as the ratio of EGFP/Tomato signals and normalized to the ratio values of the SV40 UTR sensor. Error bars, s.d. Signal intensities were averaged in ten random fields totalling approximately 500- 
1,000 cells. $* P<0.001$, Student's $t$-test. WT, wild type. d, Selective expression of miR- $9 *$ in post-mitotic neurons. Photographs show fluorescent in situ hybridization analysis of miR-9* expression (in green) and $\beta$-tubulin-III staining (in red) in the neural tubes of E11.5 embryos. 

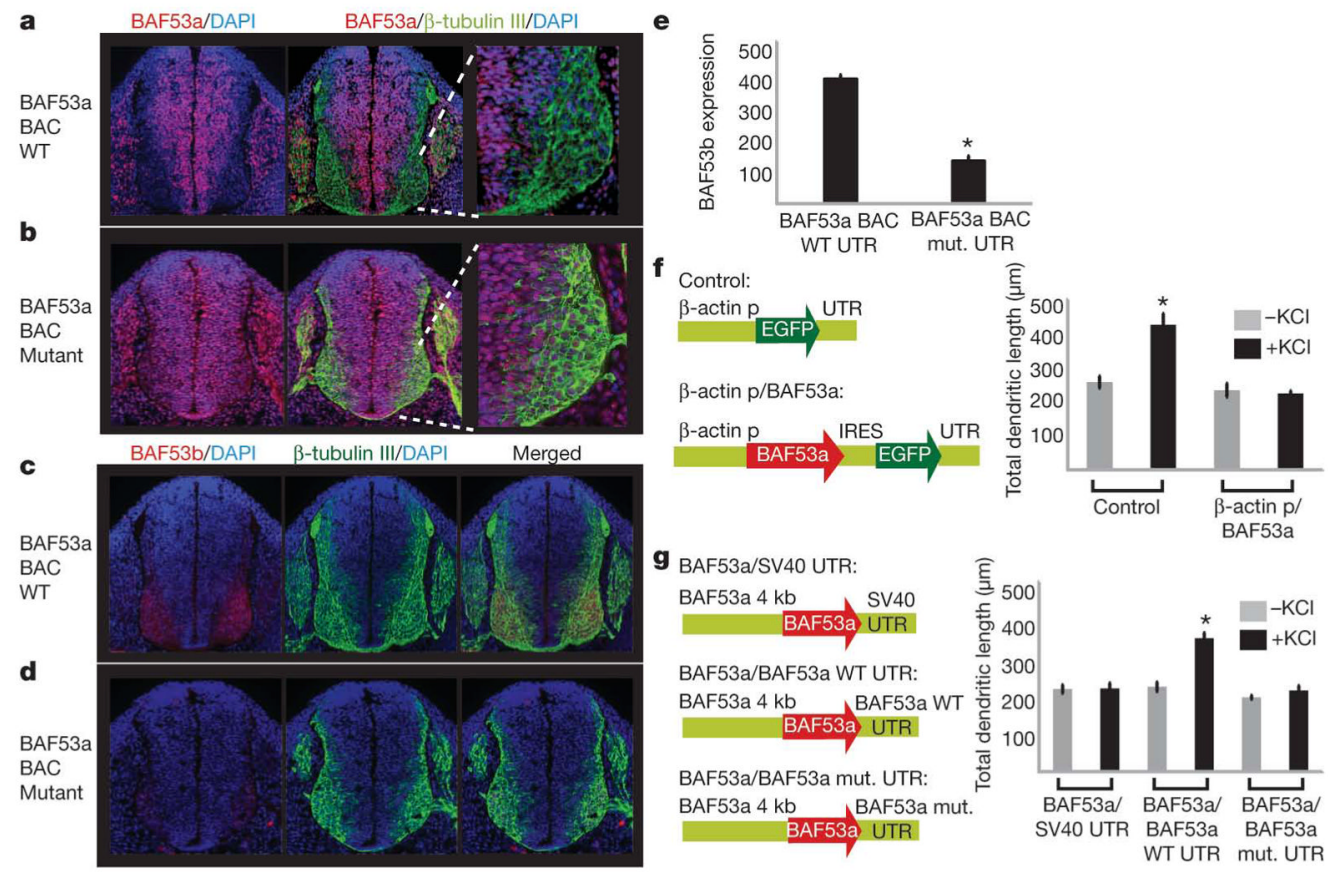

Figure 3. BAF53a repression is essential for activity-dependent dendritic outgrowth in neurons a, Normal downregulation of BAF53a in post-mitotic neurons in transgenic embryos with wild type BAF53a BAC. The rightmost panel shows the lower-right quadrant of the neural tube.

b, Persistent expression of BAF53a in neurons seen with BAF53a BAC containing point mutations in the miRNA-binding sites. $\mathbf{c}$, Normal expression of BAF53b (red) in $\beta$-tubulinIII-positive (green) neurons in transgenic embryos with wild-type BAF53a BAC. d, Reduced BAF53b expression with persistent expression of BAF53a in neurons.

e, Quantification of BAF53b expression: ratio of BAF53b level (arbitrary units) and $\beta$ tubulin-III-positive neurons. Average values are from eight sections of the neural tube. Error bars, s.e. ${ }^{*} P<0.005$, Student's $t$-test. f, Constructs to overexpress BAF53a in cultured hippocampal neurons and quantification of dendritic outgrowth of GFP-positive neurons upon stimulation using $\mathrm{KCl}$. The average values are from five individual coverslips from two independent experiments, with each coverslip containing 50-100 scored neurons. Error bars, s.e. ${ }^{*} P<0.005$, Student's $t$-test. p, promoter; IRES, internal ribosome entry site. $\mathbf{g}$, Schematic diagrams of BAF53a expression constructs using different $3^{\prime}$ UTRs and quantification of dendritic outgrowth of transfected neurons upon stimulation using $\mathrm{KCl}$. In independent experiments, we found that the 4-kb upstream region of BAF53a (illustrated) was sufficient to drive expression of GFP reporters that could be repressed by endogenous miR-9* and miR-124. Error bars, s.e. ${ }^{*} P<0.001$, Student's $t$-test. 
a

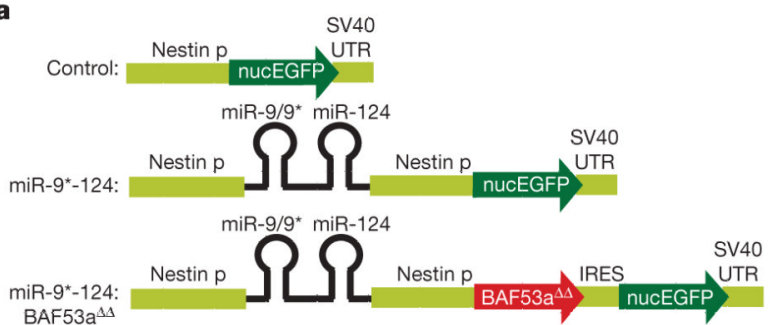

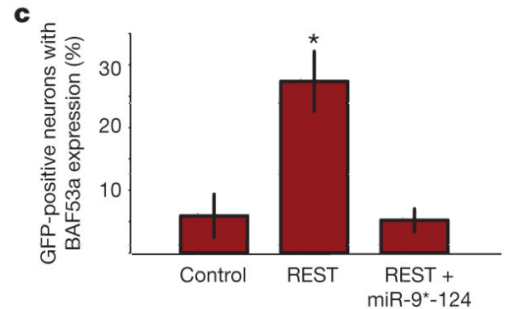

d

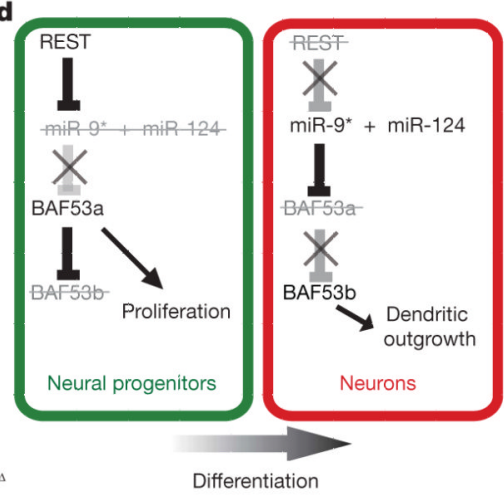

Figure 4. Effect of miR-9* and miR-124 overexpression in progenitors and REST in neurons a, miR-9* and miR-124 overexpression constructs (miR-9*-124) expressed in progenitors. nucEGFP, nuclear enhanced green fluorescent protein. $\mathbf{b}$, Proliferative defect caused by miR-9* and miR-124 overexpression in progenitors. Overexpression of miR-9* and miR-124 reduced BAF53a expression in the EGFP-positive progenitors relative to control (upper-right graph). Photographs show transgenic embryos containing either control or miR-9*-124 overexpression constructs and $\mathrm{K}_{\mathrm{i}}-67$ staining, quantified in the lower-right graph on the basis of nine individual tissue sections. Error bars, s.e. $* P<0.001$, Student's $t$ test. c, BAF53a regulation is mediated by REST. Hippocampal neurons cultured from E18.5 embryos were transfected with control (EGFP only), REST (EGFP +REST expression constructs) or REST +miR-9*-124 (EGFP +REST +miRNA overexpression constructs). The quantification represents average percentages of transfected GFP-positive neurons that had BAF53a expression from five coverslips. We analysed 15-25 GFP-positive neurons per coverslip. Error bars, s.e. $* P<0.05$, Student's $t$-test. d, Genetic pathway leading to BAF53subunit switching during neuronal differentiation. 